\title{
UN TEOREMA SULLE PROJEZIONI ORTOGONALI DI DUE SEGMENTI RETTANGOLARI E LA SUA APPLICAZIONE IN GEOMETRIA DESCRITTIVA.
}

Nota del Prof. F. P. Paternò, in Palermo.

Adunanza del ro gennajo r9o4.

Il teorema che espongo, pur riguardando una posizione particolare di segmenti rettilinei fra loro e rispetto ad un piano, non è privo di utilità in Geometria descrittiva: sia perchè quei segmenti piuttosto comunemente si riscontrano nelle figure da rappresentare, ed a quella posizione è sempre facile ricondursi; ma sopratutto perchè esso tende a stabilire una miglior dipendenza fra le projezioni di quelle figure, mediante costruzioni talvolta più semplici e dirette, da valere, in ogni caso, come verifica di altre già note.

Ed ecco il teorema :

"Se la perpendicolare comune a due segmenti rettangolari di lun"ghezze $a$ e $b$ qualsivogliano, è parallela ad un piano, saranno reciproci " $i$ due rapporti fra la differenza delle ordinate (dislivelli) rispetto a que« sto, degli estremi di ciascuno di essi $\left(\begin{array}{lll}a & 0 & b\end{array}\right)$ con la projezione orto" gonale dell'altro $\left(\begin{array}{lll}b & \circ & a\end{array}\right)$ su quel piano; poichè, rispettivamente uguali «ai rapporti $a: b \circ b: a$ de' due segmenti obbiettivi dati ».

Considerando, infatti, quel piano, p. es. come orizzontale, e riferendosi ad un altro piano perpendicolare al primo (quindi verticale) e scelto in modo che riesca anch'esso parallelo alla perpendicolare comune agli anzidetti segmenti : questi e le loro projezioni su due piani risulteranno perpendicolari alla linea di terra; e quelle verticali rispettivamente quanto il dislivello degli estremi di ciascuno di essi dal sudetto piano orizzontale. Mentre, com'è chiaro (data la ortogonalità dei piani di rife- 
rimento, non che quella dei considerati segmenti e la loro scambievole giacitura) saranno reciprocamente uguali gli angoli di que' segmenti con quei piani; e quindi eguali i rapporti di raccorciamento, rispetto al segmento originale, della projezione orizzontale (verticale) dell'uno e della projezione verticale (orizzontale) dell'altro. Epperò chiamando rispettivamente $a^{\prime}, b^{\prime}$ ed $a^{\prime \prime}, b^{\prime \prime}$ le lunghezze delle loro projezioni orizzontali e verticali si avrà

$$
\frac{a^{\prime}}{a}=\frac{b^{\prime \prime}}{b} \quad \text { ed } \quad \frac{a^{\prime \prime}}{a}=\frac{b^{\prime}}{b}
$$

d'onde

$$
\frac{a^{\prime \prime}}{b^{\prime}}=\frac{a}{b} \quad \text { ed } \frac{b^{\prime \prime}}{a^{\prime}}=\frac{b}{a}, \text { cioè } \frac{a^{\prime \prime}}{b^{\prime}}=\mathrm{I}: \frac{b^{\prime \prime}}{a^{\prime}} \text {. C. D. D. }
$$

Dalle quali relazioni, meglio sotto la forma

$$
a^{\prime \prime}=b^{\prime} \frac{a}{b} \quad \text { e } \quad b^{\prime}=a^{\prime \prime} \frac{b}{a} ; \quad b^{\prime \prime}=a^{\prime} \frac{b}{a} \quad \text { ed } \quad a^{\prime}=b^{\prime \prime} \frac{a}{b},
$$

relative al caso di due segmenti rettangolari disposti normalmente alla linea di terra, risulta evidente l'utilità del teorema, specie quanto più semplice è il rapporto fra le loro lunghezze. Giacchè, invece di dedurre l'una dall'altra le projezioni di ciascuno di essi, mediante le risapute costruzioni, si può, più facilmente, ottenere la projezione dell'uno da quella non omonima dell'altro, moltiplicando la lunghezza di quest'ultimo pel rapporto degli originali segmenti, considerati nell'ordine stesso di quelle loro projezioni : ciò che si traduce nel disegno, per mezzo di operazioni grafiche, spesso assai semplici.

Chè se quei segmenti non fossero perpendicolari alla linea di terra, ma soltanto nella posizione indicata dal teorema rispetto a un dato piano, p. es. l'orizzontale, basterebbe allora, com’è ovvio, sostituire nell'enunciato de' sudetti risultati, alle projezioni verticali, i dislivelli degli estremi di ciascuno di essi, rispetto a quel piano: dislivelli che, com'è noto, non cambiano comunque si scelga il piano verticale.

Sono, infine, degni di nota $i$ seguenti casi particolari del teorema, che più propriamente convengono a talune quistioni.

$I^{\circ}$ Se di quei segmenti rettangolari sono eguali in lunghezza le projezioni orizzontali (i dislivelli degli estremi di ciascuno di essi) il loro valor comune sarà medio geometrico fra i dislivelli degli estremi di ciascuno di essi (fra le lunghezze delle loro projezioni orizzontali); come 
risulta facendo successivamente $a^{\prime}=b^{\prime}$ ed $a^{\prime \prime}=b^{\prime \prime}$ nella relazione già trovata $\frac{a^{\prime \prime}}{b^{\prime}}=\mathrm{r}: \frac{b^{\prime \prime}}{a^{\prime}}$.

$2^{\circ}$ Se i considerati segmenti rettangolari sono d'ugual lunghezza, ne viene che il dislivello degli estremi di ciascuno di essi è quanto la projezione orizzontale dell'altro; e se sono inoltre perpendicolari entranbi alla Enea di terra, allora più semplicemente può dirsi che: la projezione orizzontale (verticale) dell'uno, è quanto quella verticale (orizzontale) dell'altro.

$3^{\circ}$ Le conclusioni del Teorema e de' suoi corollarii non cambiano se quei segmenti anzichè sghembi fossero in un piano (normale, beninteso, ad uno, almeno, de' piani di projezione): essi sarebbero, allora, i cateti di un triangolo rettangolo. E se la sua ipotenusa è perpendicolare ad uno di que' piani, viensi a ricadere, pel $\mathrm{I}^{\circ}$ caso particolare, nel noto teorema d'Euclide.

Ecco, pertanto, un breve cenno sulle applicazioni del Teorema che per amor di simmetria ne' risultati, ho preferito riferire ai poliedri regolari, ma che si potrebbero estendere, senz'altro, a tutte quelle figure con spigoli o dimensioni perpendicolari fra loro o ad un piano che vi si considera, giacchè perpendicolari allora a tutte le sue rette. E sarà manifesto, nel rappresentarli, che per passare dall'una all'altra delle loro projezioni, invece del consueto raddrizzamento di una faccia o di una sezione (metodo, questo, sempre più laborioso e indiretto) sarà molto meglio avvalersi delle facili relazioni tra le projezioni e i dislivelli (o fra le projezioni) di spigoli o diagonali rettangolari che si riscontrano in quei solidi.

a) Se un tetraedro regolare è collocato in modo, rispetto ai piani fondamentali, che l'asse di due spigoli opposti sia parallelo alla linea di terra, le sue projezioni ortogonali su guei piani risulteranno eguali (e di forma quadrata se gli altri due assi analoghi del poliedro sono rispettivamente perpendicolari ai sudetti piani di projezione).

Parimenti eguali riescono le due projezioni di un cubo, che ha quattro spigoli paralleli alla linea di terra; e cosi quelle di un ottaedro regolare, del quale una sezione quadrata, passante per quattro spigoli, è perpendicolare alla linea di terra.

E se le diagonali di tale sezione fossero inoltre perpendicolari rispettivamente ai piani di riferimento, quelle projezioni sarebbero due eguali quadrati colle loro diagonali. 
b) Dalla projezione ortogonale di un cubo sopra un piano, p. es. orizzontale, perpendicolare ad una sua diagonale (com'è noto isometrica e formata da un esagono regolare coi suoi raggi) si può subito dedurre quella verticale, avvalendosi del rapporto $\mathrm{I}: \sqrt{2}$ tra lo spigolo e la diagonale di una sua faccia. Giacchè trovandosi in esso coppie di tali elementi disposti come indica il Teorema rispetto al piano orizzontale, ne viene che l'altezza della projezione verticale (che è d'altronde quanto una diagonale del poliedro) è anche uguale alla somma de' due segmenti che si ottengono moltiplicando successivamente per $\sqrt{2}$ e per $\frac{1}{\sqrt{2}}$ la lunghezza del lato di quell'esagono: segmenti che risultando perciò l'uno doppio dell'altro, quell'altezza resta divisa in tre parti eguali dalle parallele alla linea di terra, su ciascuna delle quali cadono le projezioni verticali di tre vertici del solido.

Ed osservando, ancora, come nell'anzidetta projezione isometrica del cubo, gli spigoli e le diagonali (delle sue facce) non parallele al piano di projezione, vi si projettano egualmente lunghe, ne consegue che tale comune lunghezza (vedi $I^{\circ}$ caso particolare del Teorema) è media geometrica fra $2 / 3$ ed $1 / 3$ della diagonale del solido. Ciò può servire a passare, viceversa, dalla sua projezione verticale (sopra un piano, cioè, parallelo ad una sua diagonale) a quella isometrica.

Tenendo presente, infine, che le diagonali delle facce del cubo formano, come è noto, due eguali tetraedri regolari, nei quali le facce due a due rispettivamente parallele, riescono perpendicolari alla congiungente due de' suoi vertici opposti e la dividono in tre segmenti uguali; ne consegue che l'altezza di quei tetraedri ne è $i^{2} / 3$ : dimensione che dovrebbesi però dividere per $\sqrt{2}$, qualora gli anzidetti poliedri cubo e tetraedro avessero eguali gli spigoli. Ciò permette di assegnare, indipendentemente da risapute costruzioni, l'altezza del tetraedro regolare di dato spigolo.

Similmente, nell'ottaedro regolare, siccome ogni congiungente due vertici opposti è perpendicolare al piano della sezione quadrata che con-

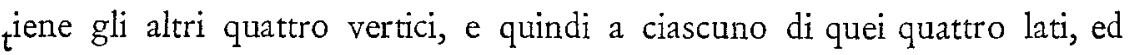
è $\sqrt{2}:$ I il rapporto fra quelle dimensioni: si passa facilmente dalla projezione ortogonale del poliedro sopra un piano parallelo a due facce opposte (projezione da considerare, p. es. come orizzontale, e che risulta di un esagono regolare colle sue diagonali minori) a quella verticale 
potendo subito assegnarne l'altezza, che è anche quanto la distanza di due facce opposte. Giacchè basta perciò moltiplicare per $\sqrt{2}$ la lunghezza del lato di quell'esagono, ovvero costruire la media geometrica fra il suo lato ed una sua diagonale maggiore.

c) Projettando, infine, ortogonalmente il dodecaedro e l'icosaedro regolari (in ciascuno dei quali sono spigoli sghembi e, nel primo, diagonali delle facce, fra loro rispettivaniente perpendicolari) sopra un piano parallelo (normale) a due facce opposte (alla congiungente due vertici opposti) sarà, quì, anche più facile dedurre da talune dimensioni di quelle projezioni (da ritenere, p. es., come orizzontali) quelle verticali; giacchè se, per maggior semplicità, si suppongano due di quegli spigoli (sghembi) rettangolari, disposti normalmente alla linea di terra, ne saranno reciprocamente uguali le projezioni di nome diverso (vedi $2^{\circ}$ caso particolare del Teorema).

Pel dodecaedro inoltre si può ancora, altrimenti, dalla sua projezione sopra un piano parallelo a due facce opposte o perpendicolare alla congiungente due vertici opposti, dedurre quelle su due piani rispettivamente perpendicolari ai primi, riferendosi ad uno dei cubi formati dalle diagonali convenientemente scelte delle sue facce: determinazioni che per altro si verificherebbero a vicenda con quelle già indicate.

Palermo, ro gennajo 1904.

F. P. Paternod. 